\title{
CARACTERIZAÇÃO DO PADRÃO DE PLANTIO ADOTADO NA ARBORIZAÇÃO DE RUAS DE CURITIBA, PARANÁ
}

\author{
Rogério Bobrowski ${ }^{1}$; Daniela Biondi ${ }^{2}$
}

\begin{abstract}
RESUMO
O plantio de árvores nas calçadas deve conciliar dois objetivos da arborização de ruas: diversidade de espécies e uniformidade de composição, visando a qualidade ambiental dos plantios e a qualidade das práticas de manejo. O objetivo desta pesquisa foi avaliar os padrões de composição de espécies utilizadas e as alterações ocorridas entre os anos de 1984 e 2010. Para isso, foram remedidas 15 unidades amostrais de um inventário realizado em 1984. Para comparação dos resultados foram analisados os padrões de plantio de cada rua e de cada parcela. As alterações completas de padrão de plantio ocorreram em três parcelas (Bacacheri 03, Boqueirão e Centro) sendo mantida a mesma composição para as demais. A análise comparativa entre os padrões de plantio e a espécie mais frequentemente plantada em cada parcela demonstrou que apenas para sete parcelas foi constatado mais que 50\% das ruas com ocorrência de espécie padrão da amostra correspondente à mais frequente, tanto em 1984 quanto em 2010. Dentre as principais espécies introduzidas como padrão de plantio destacou-se Handroanthus chrysotrichus como a opção mais usual para estabelecimento de novos padrões de plantio entre 1984 e 2010. A análise da similaridade de espécies demonstrou que a composição de espécies das parcelas manteve-se igual $(p<0,01)$, exceto para as parcelas Bacacheri 03 e Boqueirão onde foi constatada dissimilaridade de composição. Concluiu-se que por meio da definição dos padrões de plantio na arborização de ruas de Curitiba foi possível conhecer as tendências de plantio adotadas pela prefeitura municipal e que para o contexto geral amostrado não houve mudança no padrão de plantio entre os anos de 1984 e 2010.
\end{abstract}

Palavras-chave: uniformidade de composição, diversidade de composição, similaridade

\section{CHARACTERIZATION OF PLANTING PATTERN ADOPTED ON CURITIBA STREET TREES, PARANA}

\begin{abstract}
Tree planting on sidewalks should reconciliate two objectives of street trees: species diversity and composition uniformity aiming at the environmental quality of plantations and the quality of management practices. The objective of this research was to evaluate the composition patterns of species used and the changes that have occurred between the years of 1984 and 2010. For this, there were remeasured 15 sample plots of an inventory held in 1984. For results comparison, there were analyzed the planting patterns of each street and each parcel. Complete changes of planting pattern occurred in three plots (Bacacheri 03, Boqueirão and Centro) being maintained the same composition to the others. The comparative analysis between planting patterns and the most often planted specie on each plot showed that only for seven plots there was found more than 50\% of streets with occurrence of plot species pattern corresponding to the more frequent species, both in 1984 and in 2010. Among the main species introduced as planting pattern there were outstanding Handroanthus chrysotrichus as the most usual option for establishment of new planting patterns between 1984 and 2010. The analysis of species similarity showed that species composition of unit plots remained the same $(p<0.01)$, except for Bacacheri 03 and Boqueirão unit plots where were detected dissimilarity of composition. It was concluded that by defining the planting patterns on streets trees of Curitiba was possible to learn about planting trends adopted by municipal administration and that to the general context sampled there weren’t change on planting pattern between the years 1984 and 2010.
\end{abstract}

Key-words: composition uniformity, composition diversity, similarity

\footnotetext{
${ }^{1}$ Engenheiro Florestal, M.Sc., Doutorando em Engenharia Florestal pela UFPR, Departamento de Pesquisa e Monitoramento, SMMA - Av. Manoel Ribas, 2727 - Mercês - Curitiba, PR - 80810-000 - e-mail: bobrowski_roger@yahoo.com.br

${ }^{2}$ Engenheira Florestal, Dra., Departamento de Ciências Florestais da Universidade Federal do Paraná - Rua Lothário Meissner, 632 - Jardim Botânico - Curitiba, PR - 80210-170 - e-mail: dbiondi@ufpr.br
} 


\section{INTRODUÇÃO}

A preocupação com a qualidade de vida e com a compatibilização entre a conservação ambiental e o desenvolvimento econômico nas cidades tem estreita relação com os dois componentes da arborização urbana: as áreas verdes e a arborização de ruas. Segundo Bobrowski (2011) o poder público dispõe de diversas medidas técnicas para propiciar a melhoria da qualidade de vida e a conservação ambiental, sendo que dentre essas medidas está o planejamento e a implantação da arborização urbana.

Por meio do planejamento é que se obtem os benefícios almejados, desde a melhoria microclimática e redução da poluição até a contribuição com a saúde psicológica e social dos habitantes (GREY; DENEKE, 1986; SILVA FILHO et al., 2002; ESCOBEDO; ANDREU, 2008; WALTON; NOWAK e GREENFIELD, 2008; NOWAK et al., 2008). Entretanto, além dos benefícios de ordem ambiental, social e econômico, Grey e Deneke (1986) afirmaram que a arborização urbana pode proporcionar outros benefícios, com uso tanto na arquitetura (criar barreiras, solamento, ampliação ou redução de escala, criar caminhos, unificação de elementos, etc) quanto na estética paisagística (adição de linhas, formas, cores, texturas, movimento).

Ao nível de macroplanejamento da cidade, a vegetação arbórea presente nas calçadas é importante para o enriquecimento da paisagem, pois funciona como eixo estruturador espacial (orientador e identificador) e como fator de homogeneização e integração da cobertura vegetal, formando malhas no tecido urbano que correspondem ao sistema viário (BUSARELLO, 1990; MILANO, 1991).

Segundo Araújo (2008) a manutenção de padrões estéticos na cena urbana, relacionada à paisagem formada pela arborização de ruas, possui interesse difuso por relacionar-se com a qualidade de vida e com o bem-estar da população. Em função disso, deve ser planejada e conservada pelo poder público o qual deve regulamentar o sistema de arborização de ruas para propiciar o equilíbrio ambiental entre as áreas construídas e o ambiente natural alterado (SANTOS, 2001).

O uso padronizado da arborização dá as cidades características peculiares que favorece o reconhecimento delas, de suas avenidas e ruas (DANTAS; SOUZA, 2004). No caso de Curitiba, a arborização de ruas e avenidas tomadas pelo "manto verde" formado pelo entrelaçamento das copas é peculiar a diversas ruas da cidade (BOBROWSKI, 2011; LIMA NETO, 2011).

Os planos diretores da arborização de ruas de cidades brasileiras e as companhias de energia elétrica em geral têm fornecido orientações sobre os procedimentos relacionados à manutenção de padrões de plantio (RGE, 2000; COELBA, 2002; CAMPO GRANDE, 2010; COPEL, s/d), para se evitar a diluição do efeito estético proporcionado pela padronização da arborização e as condições de manejo das árvores sob fiação (BOBROWSKI; BIONDI; BAGGENSTOSS, 2009).

De acordo com RGE (2000), o programa de arborização de uma cidade deve estabelecer o padrão de plantio adotado para uma rua: se utilizará a mesma espécie para os dois lados da via ou se a composição será diversificada. Para Coelba (2002) o plantio de uma mesma espécie por trecho de rua é indicado para manter a uniformidade, mas se a via é longa seria mais conveniente alternar duas ou três espécies de um lado e de outro da rua. Segundo Castro (2010), deve-se manter a uniformidade dentro das quadras ou mesmo dentro das ruas ou avenidas utilizando uma ou até mesmo duas espécies. Para Campo Grande (2010) o padrão de plantio adotado nas ruas deve ser mantido quando das atividades de replantio. Porém, isso é dependente dos sucessos e erros observados que devem pautar a revisão do plano diretor estabelecido e a manutenção dos padrões de composição adotados.

Por outro lado, Urban Forest Commision (2011) não recomenda o uso de apenas uma ou duas espécies numa rua ou quadra porque isso aumenta consideravelmente o risco de ataque de pragas ou doenças e a possibilidade de

CARACTERIZAÇÃo DO PADRÃO... 
declínio da arborização de ruas. Desta forma, afirmaram que uma única espécie não deve exceder mais que 35\% da população arbórea de um lado da rua ou de uma quadra e não mais que 05 indivíduos arbóreos deveriam ser plantados em sequência. Entretanto, o planejamento e implantação dessa recomendação requerem cautela e sensatez, pois se deve compatibilizar a melhoria da diversidade de espécies na arborização de ruas (COELBA, 2002; BIONDI; LEAL, 2008), com os diversos benefícios possíveis e com as melhores práticas de gerenciamento da arborização, principalmente das atividades de poda que requerem uso de equipamentos automatizados (NUNES, 1995).

Desta forma, os seguintes objetivos foram formulados para a pesquisa: a) definir o padrão de plantio adotado na arborização de ruas de Curitiba; b) analisar a mudança no padrão de plantio entre os anos de 1984 e 2010; c) verificar as novas tendências adotadas como padrão de plantio.

\section{MATERIAL E MÉTODOS}

A presente pesquisa foi realizada na cidade de Curitiba, capital do Estado do Paraná, que está localizada na porção leste do Estado, no primeiro planalto paranaense entre as coordenadas $25^{\circ} 25^{\prime} 48^{\prime}$ 'S e $49^{\circ} 16^{\prime} 15^{\prime} \mathrm{O}$. A cidade está localizada em relevo levemente ondulado e altitude média igual a 934m (CURITIBA, 2011) e inserida dentro da região fitogeográfica da Floresta Ombrófila Mista que compõe o Bioma da Mata Atlântica, entremeada por pequenos fragmentos de Estepe Gramíneo-Lenhosa.

A implantação da arborização de ruas de Curitiba iniciou a partir do século XIX, sendo que somente em 1967 houve a primeira preocupação com a introdução de espécies florestais nativas (BIONDI; ALTHAUS, 2005), tais como: Handroanthus chrysotrichus (ipê-amarelomiúdo), Handroanthus albus (ipê-amarelo-graúdo), Handroanthus heptaphyllus (ipê-roxo), Tibouchina sellowiana (quaresmeira) e Poincianella pluviosa (sibipiruna). Até esta época a arborização de ruas era composta essencialmente por espécies como: Acer negundo (acer), Ligustrum lucidum (alfeneiro), Melia azedarach (cinamomo), Jacaranda mimosaefolia (jacarandá) (BIONDI; ALTHAUS, 2005).

A pesquisa foi desenvolvida sobre as mesmas unidades amostrais do inventário da arborização de ruas conduzido por Milano (1984). Para a localização e remedição das parcelas foram utilizadas cópias dos croquis de campo originais que possuíam pontos de amarração definidos e demarcados pelo inventário anterior, correspondentes a distâncias entre o eixo periférico da parcela em relação às esquinas, de cada lado das ruas avaliadas (FIGURA 01).

A coleta de dados consistiu em verificar as árvores que estavam dentro dos limites da área amostral de cada parcela, anotando-se os dados por rua avaliada. A identificação das espécies foi realizada prioritariamente "in loco", no momento da coleta de dados. Para as espécies não identificadas, ramos com folhas foram coletados, herborizados e encaminhados ao Museu Botânico da Prefeitura Municipal de Curitiba para identificação por meio de comparação com exsicatas.

Para a análise do padrão de plantio (termo adotado pela prefeitura municipal) considerou-se que o padrão seria correspondente à espécie com maior freqüência de plantio na rua avaliada, seja realizado pela prefeitura municipal ou voluntariamente pela população. Definido o padrão de plantio por rua foi realizada a definição do padrão de plantio por parcela como: a espécie mais frequentemente adotada como padrão de plantio nas ruas, ou seja, aquela espécie que mais se repetiu como padrão de plantio nas ruas avaliadas. Desta forma, foi realizada análise comparativa das alterações dos padrões de plantio entre 1984 e 2010 como indicativo de tendências de introdução de espécies adotadas pela prefeitura municipal.

O maior quantitativo de uma espécie na parcela nem sempre correspondia ao padrão de plantio, pois poderia estar restrita como padrão de uma única rua ou menor 


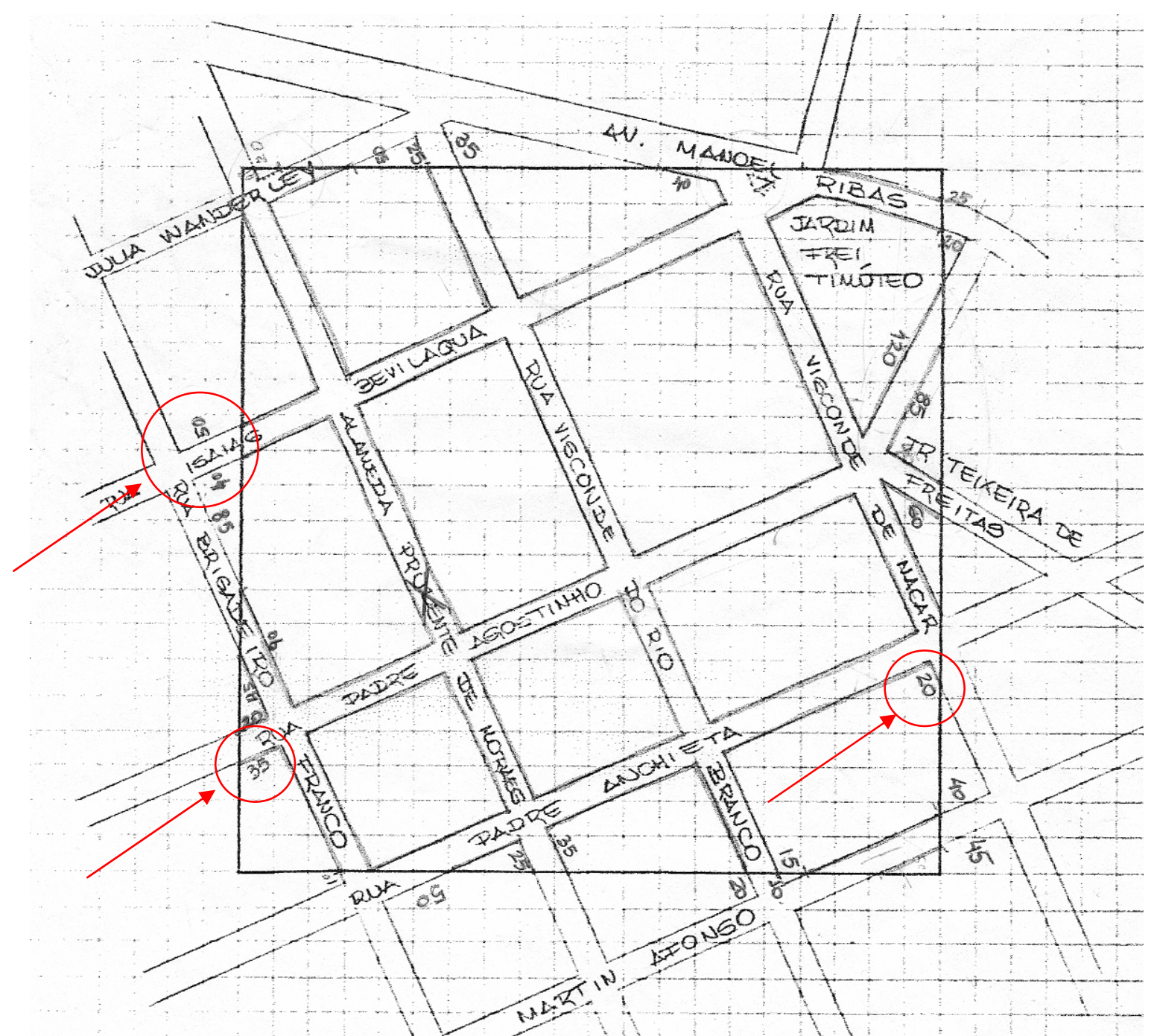

FIGURA 01 - CROQUI DE LOCALIZAÇÃO DA PARCELA MERCÊS, COM PONTOS DE AMARRAÇÃ̃ EM DESTAQUE

FIGURE 01 - SKETCH MAP OF MERCÊS PLOT LOCALIZATION WITH MOORING POINTS FEATURED

Fonte: Milano (1984)

Na análise dos resultados foi calculado o coeficiente de Jaccard (REAL; VARGAS, 1996; RODE et al., 2009) para testar a similaridade entre a composição de espécies para os pares de parcelas, de cada ano de avaliação. A significância da similaridade foi observada ao nível de $1 \%$ de probabilidade, conforme recomendações de Real (1999).

O coeficiente de Jaccard é um índice de diversidade beta utilizado para estudar a coexistência de espécies ou a similaridade entre unidades amostrais (REAL; VARGAS, 1996), sendo uma medida de correlação que varia entre 0 e 1 (RODE et al., 2009). Segundo Real e Vargas (1996) pode ser descrito pela fórmula:

$$
J=\frac{C}{A+B+C}
$$

Onde:

A - número de espécies presentes na parcela A e ausentes na parcela $\mathrm{B}$;

B - número de espécies presentes na parcela B e ausentes na parcela A;

C - número de espécies comuns entre as parcelas A e B.

CARACTERIZAÇÃo DO PADRÃO... 


\section{RESULTADOS E DISCUSSÃO}

As características dos padrões de plantio de cada parcela, em cada ano de avaliação, e as mudanças observadas para esses padrões estão representadas na Tabela 01.

As mudanças completas de padrão de plantio ocorreram nas parcelas Bacacheri 03, Boqueirão e Centro (TABELA 01). Para as duas primeiras parcelas constataram-se as seguintes mudanças: de Acer negundo (acer) para Handroanthus heptaphyllus (ipê-roxo) e Handroanthus chrysotrichus (ipê-amarelo-miúdo) na parcela Bacacheri
03, de $H$. chrysotrichus para Ligustrum lucidum (alfeneiro) na parcela Boqueirão e de Lagerstroemia indica (extremosa) para indefinido na parcela Centro. Já as alterações parciais ocorreram nas parcelas Alto da XV, com substituição de $L$. lucidum por $L$. indica, e na parcela Jardim Social, com exclusão de Handroanthus albus (ipêamarelo-graúdo). As demais parcelas mantiveram o padrão de plantio constatado em 1984.

TABELA 01 - CARACTERÍSTICAS DOS PADRÕES DE PLANTIO DA ARBORIZAÇÃO DE RUAS

\begin{tabular}{lcccccc} 
TABLE 01 - CHARACTERISTICS OF STREET TREES PLANTING PATTERNS & \\
\hline PARCELA & TRUA & TM & TP & TR & PAD 84 & PAD 10 \\
\hline Água Verde & 11 & 3 & 1 & 0 & Alf & Alf \\
Alto da XV & 11 & 3 & 0 & 0 & Alf, Acer & Acer, Ext \\
Bacacheri 01 & 10 & 1 & 2 & 0 & Ang & Ang \\
Bacacheri 02 & 9 & 1 & 0 & 0 & Ip1 & Ip1 \\
Bacacheri 03 & 12 & 3 & 7 & 0 & Acer & Ip2, Ip3 \\
Bigorrilho & 12 & 0 & 2 & 0 & Ext & Ext \\
Boqueirão & 9 & 0 & 8 & 0 & Ip3 & Alf \\
Centro & 8 & 2 & 0 & 2 & Ext & --- \\
Cristo Rei & 14 & 0 & 5 & 0 & Ip3 & Ip3 \\
Jardim Social & 14 & 4 & 1 & 0 & Ip1, Ip3 & Ip3 \\
Mercês & 11 & 0 & 0 & 0 & Alf & Alf \\
Portão & 8 & 0 & 0 & 0 & Acer & Acer \\
Rebouças 01 & 6 & 1 & 1 & 1 & Ext & Ext \\
Rebouças 02 & 9 & 0 & 0 & 1 & Ext & Ext \\
Seminário & 11 & 1 & 2 & 0 & Ext & Ext
\end{tabular}

Legenda: TRUA (número total de ruas na parcela), TM (total de ruas com mudanças de espécie mais freqüente), TP (total de ruas onde houve plantio), TR (Total de ruas onde inexistem árvores), PAD84 (padrão de plantio em maior número de ruas em 1984), PAD10 (padrão de plantio em maior número de ruas em 2010), Alf (alfeneiro), Acer (Acer), Ext (extremosa), Ang (Angico), Ip1 (ipê-amarelo-graúdo - Handroanthus albus), Ip2 (ipê-roxo - Handroanthus heptaphyllus), Ip3 (Ipê-amarelo-miúdo Handroanthus chrysotrichus)

Nas parcelas Bacacheri 03 e Boqueirão a mudança total de padrão de plantio está associada às maiores proporções de total de ruas com novos plantios, sendo respectivamente iguais a 58,33\% e $88,89 \%$. Para a parcela Centro observou-se que a inexistência de um padrão definido em 2010 está associada a um padrão de plantio diferente para cada uma das quatro ruas onde existiam árvores.

A mudança de padrão de uma espécie exótica para duas nativas na parcela Bacacheri 03 aparentemente demonstra a adoção e manutenção da regularidade dos plantios efetivados pela prefeitura municipal, porém com o uso de espécies nativas já utilizadas em 1984. Neste sentido,
Bobrowski (2011) afirmou que falta investimento em pesquisa para utilização de espécies nativas na arborização de ruas, porém o trabalho desenvolvido por Biondi e Leal (2009), pioneiro na análise da produção de mudas destinadas especificamente para a arborização de ruas, demonstrou ser possível a obtenção de resultados efetivos.

Por outro lado, a mudança de padrão de uma espécie nativa para uma espécie exótica na parcela Boqueirão provavelmente está associada aos plantios realizados voluntariamente pela população. De acordo com Silva Filho e Bortoleto (2005) e Sucomine e Sales (2010) as iniciativas particulares de plantios na arborização de ruas Rogério Bobrowski et al.. 
geram um aspecto irregular da mesma, pois introduzem espécies diferentes e muitas vezes inadequadas, potencializando problemas e prejuízos. Salienta-se que este fato é comum em cidades brasileiras, principalmente devido à falta de planejamento e gestão da arborização de ruas, sendo observado em cidades como Sete de Setembro - RS (COLETTO; MÜLLER; WOLSKI, 2008), Uchôa - SP (STRANGHETTI; SILVA, 2010), Goiandira - GO (PIRES et al., 2010) e Colorado - RS (RABER; REBELATO, 2010), porém, mesmo em cidades com arborização de ruas planejadas como Maringá - PR a ocorrência de plantios irregulares também é comum (SAMPAIO; DE ANGELIS, 2008) devido à intervenção voluntária da população.

A mudança de um padrão definido para um indefinido na parcela Centro indica a desordem da arborização das ruas, pois existe em cada rua uma espécie diferente ou a inexistência de árvores, na maioria delas. Este fato é justificado pela maior pressão e impactos sobre as árvores implantadas, associada à falta de planejamento da gestão pública para reposição das árvores removidas. Isso afetou o reconhecimento de um padrão de plantio para a parcela. Por fim, constatou-se que a maior ocorrência de mudança de espécie mais frequente, observado para as parcelas Água Verde, Alto da XV e Jardim Social, não influenciou a manutenção do padrão de plantio das mesmas.

Para melhor compreensão dos resultados obtidos foi elaborada a Tabela 02 que apresenta a análise da relação entre a espécie definida com padrão de plantio da parcela (aquela que mais se repete como padrão de plantio entre as ruas avaliadas) e a espécie mais frequentemente plantada (maior proporção total) em cada parcela.

Apenas para sete parcelas foi constatado mais que 50\% das ruas com ocorrência de espécie padrão da amostra, tanto em 1984 quanto em 2010. Para as demais parcelas foi observado que a espécie padrão da amostra ocorre em menor proporção de ruas. Isso mostra relação com a diversidade de espécies plantadas que influenciou na observação de padrão de plantio diferente para cada rua amostrada.

TABELA 02 - RELAÇÃO ENTRE ESPÉCIE PADRÃO DE PLANTIO E ESPÉCIE MAIS FREQUENTE EM UMA PARCELA

TABLE 02 - RELATIONSHIP BETWEEN SPECIE PLANTING PATTERN AND MORE FREQUENT SPECIE ON A

\begin{tabular}{|c|c|c|c|c|c|c|c|}
\hline PARCELAS & TRA & PAD(84) & PAD(10) & TREP & \%TREP & EMFP(84) & EMFP(10) \\
\hline Água Verde & 11 & Alf & Alf & $3 / 3$ & $27 / 27$ & Alf & Alf \\
\hline Alto XV & 11 & Alf, Acer & Acer, Ext & $3 / 3$ & $27 / 27$ & Ext & Ext \\
\hline Bacacheri 01 & 10 & Ang & Ang & $6 / 6$ & $60 / 60$ & Ang & Ang \\
\hline Bacacheri 02 & 9 & Ip1 & Ip1 & $8 / 7$ & $89 / 78$ & Ip1 & Ip1 \\
\hline Bacacheri 03 & 12 & Acer & Ip2, Ip3 & $2 / 3$ & $17 / 25$ & Acer & Ip2 \\
\hline Bigorrilho & 12 & Ext & Ext & $6 / 6$ & $50 / 50$ & Ext & Ext \\
\hline Boqueirão & 9 & Ip3 & Alf & $1 / 3$ & $11 / 33$ & Ip3 & Ded \\
\hline Centro & 8 & Ext & - & $4 / 0$ & $50 / 00$ & Ext & Sib \\
\hline Cristo Rei & 14 & Ip3 & Ip3 & $4 / 5$ & 29/36 & Ip3 & Tip \\
\hline Jardim Social & 14 & Ip1, Ip3 & Ip3 & $3 / 5$ & $21 / 36$ & Ip3 & Ip3 \\
\hline Mercês & 11 & Alf & Alf & $6 / 6$ & $55 / 55$ & Alf & Alf \\
\hline Portão & 8 & Acer & Acer & $3 / 3$ & $38 / 38$ & Acer & Cas \\
\hline Rebouças 01 & 6 & Ext & Ext & $3 / 3$ & $50 / 50$ & Ext & Ext \\
\hline Rebouças 02 & 9 & Ext & Ext & $5 / 5$ & $56 / 56$ & Ext & Ext \\
\hline Seminário & 11 & Ext & Ext & $6 / 7$ & $55 / 64$ & Ext & Ext \\
\hline
\end{tabular}

Legenda: TRA (total de ruas na amostra); PAD (84)(espécie padrão de plantio na amostra no ano de 1984); PAD (10)(espécie padrão de plantio na amostra no ano de 2010); TREP (total de ruas onde ocorre a espécie padrão - seja: 1984/2010); EMFP (84) (espécies com maior frequência de plantio no ano de 1984); EMFP (10) (espécies com maior frequência de plantio no ano de 2010); Alf (Alfeneiro - Ligustrum lucidum), Acer (Acer - Acer negundo), Ext (Extremosa - Lagerstroemia indica), Ang (Angico - Parapiptadenia rigida), Ip1 (ipê-amarelograúdo - Handroanthus albus), Ip2 (ipê-roxo - Handroanthus heptaphyllus), Ip3 (Ipê-amarelo-miúdo - Handroanthus chrysotrichus); Ded (Dedaleiro - Lafoensia pacari); Sib (Sibipiruna - Poincianella pluviosa); Tip (Tipuana - Tipuana tipu); Cas (Falso-barbatimão - Cassia leptophylla)

Por outro lado, a relação entre a espécie com maior frequência de plantio e a espécie padrão de plantio da amostra foi observada para 11 parcelas, para ambos os anos de avaliação. Como já citado anteriormente, o maior

CARACTERIZAÇÃO DO PADRÃO... 
quantitativo de uma espécie na parcela, pode não ser o padrão de plantio, pois pode estar restrito como padrão de uma única rua ou menor número de ruas do que outra espécie que se repete como padrão de várias ruas, fato este constatado para as parcelas Alto da XV, Cristo Rei e Portão.

A Tabela 03 apresenta informações sobre as parcelas onde foi constatada a introdução de espécies como padrão de plantio em 2010, em relação à amostragem de 1984.
São descritas as espécies introduzidas, as parcelas afetadas e o porte da espécie utilizada.

A análise dessa tabela demonstra que cinco espécies se destacaram como as principais espécies introduzidas, pois foram adotadas como padrão de plantio em ruas de três ou mais parcelas. Destas espécies destaca-se $H$. chrysotrichus como a opção mais usual para estabelecimento de novos padrões de plantio entre 1984 e 2010, pois foi plantada como padrão em ruas de seis das quinze parcelas avaliadas.

TABELA 03 - PARCELAS ONDE FOI CONSTATADA A INTRODUÇÃO DE ESPÉCIES COMO PADRÃO DE PLANTIO EM 2010, EM RELAÇÃO À AMOSTRAGEM DE 1984 TABLE 03 - UNIT PLOTS WHERE WERE INTRODUCED SPECIES LIKE PLANTING PATTERN IN 2010, IN RELATION TO 1984 SAMPLING

\begin{tabular}{llc}
\hline ESPÉCIES INTRODUZIDAS & \multicolumn{1}{c}{ PARCELAS } & PORTE \\
\hline Lagerstroemia indica & AV, AX, CR, R1, SE & $\mathrm{P}$ \\
Poincianella pluviosa var. peltophoroides & $\mathrm{AV}, \mathrm{BO}$ & $\mathrm{M}$ \\
Handroanthus chrysotrichus & $\mathrm{AV}, \mathrm{B} 2, \mathrm{~B} 3, \mathrm{CR}, \mathrm{JS}, \mathrm{SE}$ & $\mathrm{M}$ \\
Acer negundo & $\mathrm{AV}, \mathrm{CR}$ & $\mathrm{P}$ \\
Hibiscus rosa-sinensis & $\mathrm{AX}, \mathrm{B} 1, \mathrm{SE}$ & $\mathrm{G}$ \\
Melia azedarach & $\mathrm{B} 1$ & $\mathrm{M}$ \\
Lafoensia pacari & $\mathrm{B} 1, \mathrm{~B} 3, \mathrm{BO}$ & $\mathrm{G}$ \\
Handroanthus heptaphyllus & $\mathrm{B} 3$ & $\mathrm{P}$ \\
Schinus molle & $\mathrm{B} 3$ & $\mathrm{M}$ \\
Ligustrum lucidum & $\mathrm{B} 3, \mathrm{BO}, \mathrm{CR}$ & $\mathrm{G}$ \\
Libidibia ferrea var. leiostachya & $\mathrm{BG}$ & $\mathrm{P}$ \\
Tibouchina pulchra & $\mathrm{BG}$ & $\mathrm{G}$ \\
Grevillea robusta & $\mathrm{BO}$ & $\mathrm{G}$ \\
Persea americana & $\mathrm{BO}$ & $\mathrm{G}$ \\
Populus nigra & $\mathrm{CT}$ & $\mathrm{M}$ \\
Handroanthus umbellatus & $\mathrm{CT}$ & $\mathrm{G}$ \\
Eucalytus saligna & $\mathrm{CR}$ & $\mathrm{G}$ \\
Tipuana tipu & $\mathrm{JS}$ & $\mathrm{G}$ \\
Araucaria angustifolia & $\mathrm{JS}$ & $\mathrm{P}$ \\
Leucaena leucocephala & $\mathrm{R} 1$ &
\end{tabular}

Legenda: Bairros: AV (Água Verde), AX (Alto da XV), B1 (Bacacheri 01), B2 (Bacacheri 02), B3 (Bacacheri 03), BG (Bigorrilho), BO (Boqueirão), CR (Cristo Rei), CT (Centro), JS (Jardim Social), R1 (Rebouças 01)

${ }^{1}$ De acordo com Lorenzi (2000), Lorenzi et al. (2003) e Biondi e Althaus (2005)

Entretanto, observa-se a utilização frequente de $L$. indica como opção, seguida de Hibiscus rosa-sinensis (hibisco) e Lafoensia pacari (dedaleiro). Ressalta-se que tais espécies são de médio porte, com exceção de $H$. rosasinensis que é de pequeno porte e arbustiva conduzida como arbórea. Evidencia-se, então, a preferência pelas mesmas, como tentativa de conciliar os benefícios almejados pela implantação da arborização das ruas com a redução de problemas, prejuízos e conflitos com as estruturas urbanas. Todavia, em espécies como $H$. chrysotrichus, $L$. indica e $H$. rosa-sinensis sobressaem-se muito mais os benefícios estéticos pela beleza da floração do que benefícios ambientais como a redução da amplitude térmica diária, redução da poluição sonora e atmosférica e redução do escoamento superficial pluvial, pois suas copas são menores e com folhagem menos densa.

Extremosa e alfeneiro são comuns dentre as principais espécies da arborização de ruas nos Estados do Sul do Brasil, tendo sido observadas em avaliações de cidades 
como Pato Branco - PR (SILVA et al., 2007), Irati - PR (COLTRO; MIRANDA, 2007), Sete de Setembro - RS (COLETTO; MÜLLER; WOLSKI, 2008), São José do Cerrito - SC (PINHEIRO et al., 2009), Frederico Westphalen - RS (BAAL; MANTOVANI, 2010), Guatambu - SC (BOHNER et al., 2010) e Santo Antônio da Patrulha - RS (BORBA; FALKOSKI; SILVA, 2010). A permanência de alfeneiro como padrão de plantio em ruas da cidade de Curitiba foi constatada em virtude de sua grande utilização em plantios mais antigos, mas que atualmente tem sido objeto de substituição gradativa, tendo em vista a política ambiental do município e o caráter invasor da espécie (BOBROWSKI, 2011).

Apesar de ser uma espécie exótica, mas de carater não invasor na cidade de Curitiba, $L$. indica ainda é utilizada por ser uma das poucas opções para uso sob fiação de transmissão de energia elétrica, em função de suas características de pequeno porte e pequena dimensão de copa, além do efeito estético causado pela floração (BOBROWSKI, 2011).

Algumas espécies observadas como padrão de plantio correspondem a espécies presentes em pequenos trechos de ruas avaliadas (canto de amostra) ou a ruas com pequena quantidade de árvores onde o padrão observado correspondia a plantios irregulares (aqueles não realizados pela prefeitura municipal). Dentre as espécies nesta condição são citadas: Schinus molle (aroeira-salsa), Grevillea robusta (grevílea) e Eucalytus saligna (eucalipto), sendo as duas últimas caracterizadas como de grande porte.

As espécies Araucaria angustifolia (araucária) e Leucaena leucocephala (leucena) são espécies introduzidas pela prefeitura municipal como padrão de plantio em dois canteiros centrais distintos, da Av. Nossa Senhora da Luz e da Av. Silva Jardim, respectivamente. Nesse contexto, vale destacar ainda a introdução de Libidibia ferrea var. leiostachya (pau-ferro) como padrão de plantio nos canteiros da canaleta do biarticulado presente na Av. Padre Anchieta e de Poincianella pluviosa var. peltophoroides (sibipiruna) como padrão de plantio no canteiro contínuo da canaleta do biarticulado em um trecho da Av. Marechal Floriano Peixoto.

A similaridade de espécies entre os dois inventários, analisada pelo índice de Jaccard, é apresentada na Tabela 04.

TABELA 04 - ÍNDICE DE JACCARD PARA OS INVENTÁRIOS DE 1984 E 2010 TABLE 04 - JACCARD INDEX TO THE INVENTORIES OF 1984 AND 2010

\begin{tabular}{lcccc}
\hline PARCELA & EU 84 & EU 10 & EC & JACCARD \\
\hline Água Verde & 13 & 17 & 22 & $0,423^{* *}$ \\
Alto da XV & 4 & 14 & 8 & $0,308^{* *}$ \\
Bacacheri 01 & 11 & 13 & 11 & $0,314^{* *}$ \\
Bacacheri 02 & 16 & 25 & 10 & $0,196^{* *}$ \\
Bacacheri 03 & 3 & 49 & 8 & $0,133^{\text {ns }}$ \\
Bigorrilho & 6 & 20 & 17 & $0,395^{* *}$ \\
Boqueirão & 1 & 28 & 2 & $0,065^{\text {ns }}$ \\
Centro & 2 & 7 & 5 & $0,357^{* *}$ \\
Cristo Rei & 10 & 27 & 14 & $0,275^{* *}$ \\
Jardim Social & 17 & 27 & 27 & $0,380^{* *}$ \\
Mercês & 7 & 14 & 19 & $0,475^{* *}$ \\
Portão & 6 & 15 & 8 & $0,276^{* *}$ \\
Rebouças 01 & 2 & 6 & 3 & $0,273^{* *}$ \\
Rebouças 02 & 7 & 18 & 11 & $0,306^{* *}$ \\
Seminário & 28 & 7 & 17 & $0,327^{* *}$ \\
\hline Total & $\mathbf{2 7}$ & $\mathbf{5 6}$ & $\mathbf{6 6}$ & $\mathbf{0 , 4 4 3} *$ \\
\hline
\end{tabular}

Legenda: EU 84 = espécies únicas de 1984; EU 10 = espécies únicas de 2010; EC = espécies em comum; ** = similaridade estatisticamente significativo $(p<0,01)$; ns = similaridade não significativa $(p>0,01)$.

A análise deste coeficiente demonstrou haver diferença estatisticamente significativa $(p<0,01)$ para 0 total amostrado de cada ano de avaliação e para treze das quinze parcelas amostradas. Isto demonstra que foi

CARACTERIZAÇÃO DO PADRÃO... 
mantida a mesma composição de espécies na arborização analisada.

A inexistência de significância estatística para as parcelas Bacacheri 03 e Boqueirão demonstra a dissimilaridade entre os pares de parcelas dos anos de 1984 e 2010. Este fato explica a caracterização dos pares de parcelas como de padrão de composição diferentes, ao contrário dos demais pares de parcelas que mantiveram o mesmo padrão de composição de espécies no intervalo de 26 anos entre as duas avaliações.

\section{CONCLUSÕES}

Foi possível identificar o padrão de plantio adotado na arborização de ruas de Curitiba, tanto para as ruas quanto para as parcelas avaliadas. A análise das espécies padrões permitiu conhecer as tendências de plantio adotadas pela prefeitura municipal, tendo havido o incremento no plantio de espécies nativas, tais como: Lafoensia pacari (dedaleiro), Libidibia ferrea (pau-ferro), Handroanthus chrysotrichus (ipê-amarelo-miúdo) e Handroanthus heptaphyllus (ipê-roxo).
Para o contexto geral amostrado não houve mudança no padrão de plantio entre os anos de 1984 e 2010, tendo em vista que as alterações completas ocorreram em parcelas onde houve maior proporção de total de ruas com novos plantios.

Há indícios de tendência de introdução de espécies como $H$. chrysotrichus, L. indica, L. pacari e H. rosa-sinensis, que se incrementados os plantios podem favorecer a alteração dos padrões de plantio das parcelas avaliadas.

\section{REFERÊNCIAS BIBLIOGRÁFICAS}

ARAUJO, E. C. Preservação ambiental de cidades: uma tradução jurídica e urbanística do Estatuto da Cidade. Cadernos Metrópole, v. 19, p. 67-79, 2008.

BAAL, F. B.; MANTOVANI, N. Arborização urbana no município de Frederico Westphalen - o problema da compatibilidade. In: Congresso Brasileiro de Arborização Urbana, 14., 2010, Bento Gonçalves. Anais..., Bento Gonçalves: SBAU, 2010. p. 169-179

BIONDI, D.; ALTHAUS, M. Árvores de rua de Curitiba: cultivo e manejo. Curitiba: FUPEF, 2005.

BIONDI, D.; LEAL, L. Caracterização das plantas produzidas no Horto Municipal da Barreirinha, Curitiba/PR. Revista da Sociedade Brasileira de Arborização Urbana, Piracicaba, v. 03, n. 02, p. 20-36. 2008.

BIONDI, D.; LEAL, L. Comportamento silvicultural de espécies nativas em viveiro de espera para uso potencial em arborização de ruas. Scientia Forestalis, Piracicaba, v. 37, n. 83, p. 313-319. 2009.

BOBROWSKI, R.; BIONDI, D.; BAGGENSTOSS, D. Composição de canteiros na arborização de ruas de Curitiba (PR). Revista da Sociedade Brasileira de Arborização Urbana, Piracicaba, v. 04, n. 02, p. 44-61. 2009.

BOBROWSKI, R. Estrutura e dinâmica da arborização de ruas de Curitiba, Paraná, no período 1984 - 2010. $144 f$. Dissertação (Mestrado em Engenharia Florestal) - Setor de Ciências Agrárias, Universidade Federal do Paraná, Curitiba, 2011.

BOHNER, T. O. L.; GRACIOLI, C. R.; WITECK NETO, L.; SOARES, K. P.; CALLEGARO, R. M.; ANDRZEJEWSKI, C. Avaliação da composição florística, porte e estado fitossanitário das árvores urbanas no município de Guatambú - SC. In: Congresso Brasileiro de Arborização Urbana, 14., 2010, Bento Gonçalves. Anais..., Bento Gonçalves: SBAU, 2010.

BORBA, M. S.; FALKOSKI, J. R.; SILVA, A. G. Inventário arbóreo quali-quantitativo na área urbana do município Santo Antônio da Patrulha, Rio Grande do Sul, Brasil. In: Congresso Brasileiro de Arborização Urbana, 14., 2010, Bento Gonçalves. Anais..., Bento Gonçalves: SBAU, 2010. 
BUSARELLO, O. Planejamento urbano e arborização. In: Encontro Nacional sobre Arborização Urbana, 3., 1990, Curitiba. Anais..., Curitiba: FUPEF, 1990. 54-59p.

CAMPO GRANDE, Prefeitura Municipal. Plano diretor de arborização urbana de Campo Grande. Campo Grande: SEMADUR, 2010. Disponível em:< www.capital.ms.gov.br/egov/downloadFile.php?id=3103>. Acesso em: 27/06/2011.

CASTRO, A. Plano de Arborização Urbana. CONDEMASAA: Santo Antônio do Aracanguá, 15p. 2010. Disponível em: $<$ http://www.saaracangua.sp.gov.br/upload/files/PlanodeArborizacao\%20Urbana.pdf>. Acesso em: 15/04/2011.

COELBA - COMPANHIA DE ELETRICIDADE DO ESTADO DA BAHIA. Guia de arborização urbana. Salvador: Coelba, 2002.

COLETTO, E. P.; MÜLLER, N. G.; WOLSKI, S. S. Diagnóstico da arborização das vias públicas do município de Sete de Setembro-RS. Revista da Sociedade Brasileira de Arborização Urbana, Piracicaba, v. 03, n. 02, p.110-122, 2008.

COLTRO, E. M.; MIRANDA, G. M. Levantamento da arborização urbana pública de Irati - PR e sua influência na qualidade de vida de seus habitantes. Revista Eletrônica Lato Sensu, v. 02, n. 01, p. 27-48, 2007. Disponível em: <http://web03.unicentro.br/especializacao/Revista_Pos/P\%C3\%A1ginas/2\%20Edi\%C3\%A7\%C3\%A3o/Engenharia/PDF/8Ed2_EN-LevArbo.pdf>. Acesso em 13/02/2011.

COPEL, Companhia Paranaense de Energia Elétrica. Arborização de Vias Públicas: Guia para os Municípios. Curitiba, s/d.

DANTAS, I. C.; SOUZA, C. M. C. Arborização urbana na cidade de Campina Grande - PB: inventário e suas espécies. Revista de Biologia e Ciências da Terra, Campina Grande, v. 04, n. 02, s/p, 2004.

ESCOBEDO, F.; ANDREU, M. A community guide to Urban Forest Inventories. Florida: University of Florida, Institute of Food and Agricultural Sciences (IFAS), 2008. 4p. Disponível em: <http://edis.ifas.ufl.edu/pdffiles/FR/FR23200.pdf>. Acesso em: 21/06/2009.

GREY, G. W.; DENEKE, F. J. Urban Forestry. 2.ed. New York: J. Wiley, 1986. 199p.

LORENZI, H. Árvores Brasileiras: manual de identificação e cultivo de plantas arbóreas do Brasil. Nova Odessa: Plantarum, v. 01, 3ª ed., 352p. 2000

LORENZI, H.; SOUZA, H. M.; TORRES, M. A. V.; BACHER, L. B. Árvores exóticas no Brasil: madeireiras, ornamentais e aromáticas. Nova Odessa: Plantarum, 368p. 2003.

LIMA NETO, E. M. Aplicação do Sistema de Informações Geográficas para o inventário da arborização de ruas de Curitiba, PR. 108f. Dissertação (Mestrado em Engenharia Florestal) - Setor de Ciências Agrárias, Universidade Federal do Paraná, Curitiba, 2011.

MILANO, M. S. Curso sobre Arborização Urbana. Curitiba: FUPEF, 1991. 75p.

NOWAK, D. J.; WALTON, J. T.; STEVENS, J. C.; CRANE, D. E.; HOEHN, R. E. Effect of plot and sample size on timing and precision of Urban Forest Assessments. Arboriculture \& Urban Forestry, Champaign, IL, v. 34, n. 06, p.386-390, 2008.

NUNES, M. L. Avaliação das necessidades de manejo e compatibilidade entre a arborização de ruas e redes de energia em Apucarana e Cascavel - Paraná. 104f. Dissertação (Mestrado em Engenharia Florestal) - Setor de Ciências Agrárias, Universidade Federal do Paraná, Curitiba, 1995.

PINHEIRO, R.; FRANCHIN, E.; RIBEIRO, R. S.; WOLFF, W; SILVA, A. C.; HIGUCHI, P. Arborização urbana na cidade de são José do Cerrito, SC: diagnóstico e proposta para ares de maior trânsito. Revista da Sociedade Brasileira de Arborização Urbana, Piracicaba, v. 04, n. 04, p.63-78, 2009.

PIRES, N. A. M. T.; MELO, M. S.; OLIVEIRA, D. A.; XAVIER-SANTOS, S. A arborização urbana do município de Goiandira/GO - caracterização quali-quantitativa e proposta de manejo. Revista da Sociedade Brasileira de Arborização Urbana, Piracicaba, v. 05, n. 03, p.185-205, 2010.

RABER, A. P.; REBELATO, G. S. Arborização viária no município de Colorado, RS - Brasil: análise quali-quatitativa. Revista da Sociedade Brasileira de Arborização Urbana, Piracicaba, v. 05, n. 01, p.193-199, 2010.

CARACTERIZAÇÃo DO PADRÃO... 
REAL, R.; VARGAS, J. M. The Probabilistic Basis of Jaccard's Index of Similarity. Systematic Biology, Oxford, v. 45, n. 03, p. 380-385,1996.

REAL, R. Tables of significant values of Jaccard's index of similarity. Miscellània Zoològica, Barcelona, v. 22, n. 01, p. 2940,1999.

RGE - RIO GRANDE ENERGIA. Manual de arborização e poda. 2000, 39p.

RODE, R.; FIGUEIREDO FILHO, A.; GALVÃO,F.; MACHADO, S. A. Comparação florística entre uma floresta ombrófila mista e uma vegetação arbórea estabelecida sob um povoamento de Araucaria angustifolia de 60 anos. Revista Cerne, Lavras, v. 15, n.01, p. 101-115, 2009.

SAMPAIO, A. C. F.; DE ANGELIS, B. L. D. Inventário e análise da arborização de vias públicas de Maringá-PR. Revista da Sociedade Brasileira de Arborização Urbana, Piracicaba, v. 03, n. 01, p.37-57, 2008.

SANTOS, A. S. R. Arborização urbana: importância e proteção. Revista Meio Ambiente Industrial, ano VI, Edição 34, n 33, 2001. 40-41p.

SILVA FILHO, D. F.; PIZETTA, P. V. C.; ALMEIDA, J. B. S. A.; PIVETTA, K. F. L.; FERRAUDO, A. S. Banco de dados relacional para cadastro, avaliação e manejo da arborização em vias públicas. Revista Árvore, Viçosa, v. 26, n. 05, p.629642, 2002.

SILVA FILHO, D. F.; BORTOLETO, S. Uso de indicadores de diversidade na definição de plano de manejo da arborização viária de Águas de São Pedro-SP. Revista Árvore, Viçosa, v. 29, n. 06, p.973-982, 2005.

SILVA, L. M.; MOCCELLIN, R.; WEISSHEIMER, D. I.; ZBORALSKI, A. R.; FONSECA, L.; RODIGUIERO, D. A. Inventário e sugestões para arborização de via pública de Pato Branco/PR. Revista da Sociedade Brasileira de Arborização Urbana, Piracicaba, v. 02, n. 01, p.101-108, 2007.

STRANGHETI, V.; SILVA, Z. A. V. Diagnóstico da arborização das vias públicas do município de Uchoa-SP. Revista da Sociedade Brasileira de Arborização Urbana, Piracicaba, v. 05, n. 02, p.124-138, 2010.

SUCOMINE, N. M.; SALES, A. Caracterização e análise do patrimônio arbóreo da malha viária urbana central do município de São Carlos-SP. Revista da Sociedade Brasileira de Arborização Urbana, Piracicaba, v. 05, n. 04, p.126-137, 2010.

URBAN FORESTRY COMMISSION. City of Reno Approved Street Tree Species List. Reno, NV, 2011. 8p. Disponível em: <www.reno.gov/Modules/ShowDocument.aspx?documentid=28407> Acesso em: 22/03/2011.

WALTON, J. F.; NOWAK, D. J.; GREENFIELD, E. J. Assessing Urban Forest Canopy cover using airbone or satellite imagery. Arboriculture \& Urban Forestry, Champaign, IL, v. 34, n. 06, p.334-340, 2008. 